\title{
NEW INEQUALITIES OF WIRTINGER TYPE FOR CONVEX AND $M N$-CONVEX FUNCTIONS
}

\author{
Tatjana Z. Mirković
}

(c) 2019 by University of Niš, Serbia | Creative Commons Licence: CC BY-NC-ND

Abstract. In this paper, we obtain some inequalities of Wirtinger type by using some classical inequalities and means for convex functions and establish some applications to special means for positive real numbers.

Keywords. Inequalities; inequalities of Wirtinger type; convex functions.

\section{Introduction}

Let $f$ be a periodic function with period $2 \pi$ and let $f^{\prime} \in L^{2}$. Then, if $\int_{0}^{2 \pi} f(x) d x=$ 0 , the following inequality holds

$$
\int_{0}^{2 \pi} f^{2}(x) d x \leqslant \int_{0}^{2 \pi} f^{\prime 2}(x) d x
$$

with equality if and only if $f(x)=A \cos x+B \sin x$, where $A$ and $B$ are constants.

Inequality (1.1) is known in the literature as Witinger's inequality. The proof of W. Wirtinger was first published in 1916 in the book (see [1]) by W. Blaschke. There are many studies which generalize and extend Wirtinger's inequality in the literature, (see [2], [3]). However, Inequality (1.1) was known before this, though with other conditions on the function $f$. For example, in 1905, E.Almansi proved that

$$
\int_{a}^{b} f^{2}(x) d x \leqslant\left(\frac{b-a}{2 \pi}\right)^{2} \int_{a}^{b}\left[f^{\prime}(x)\right]^{2} d x,
$$

Received August 14, 2018; Accepted November 28, 2018

2010 Mathematics Subject Classification. Primary 26A51; Secondary 26D10 
under the condition that $f$ and $f^{\prime}$ are continuous on the interval $(a, b)$, that $f(a)=$ $f(b)$ and that $\int_{a}^{b} f(x) d x=0$.

Theorem 1.1. (Hölder inequality) Let $f(x)$ and $g(x)$ be positive continuous functions on $[a, b]$. If $p>1$ and $\frac{1}{p}+\frac{1}{q}=1$, then

$$
\int_{a}^{b} f(x) g(x) d x \leqslant\left(\int_{a}^{b} f(x)^{p} d x\right)^{\frac{1}{p}}\left(\int_{a}^{b} g(x)^{q} d x\right)^{\frac{1}{q}} .
$$

Theorem 1.2. (Reverse Hölder inequality) For two positive functions $f$ and $g$ satisfying $0<m \leqslant \frac{f^{p}}{g^{q}} \leqslant M<\infty$, on the set $X$, and for $p, q>1$ with $\frac{1}{p}+\frac{1}{q}=1$, we get

$$
\left(\int_{X} f^{p} d \mu\right)^{\frac{1}{p}}\left(\int_{X} g^{q} d \mu\right)^{\frac{1}{q}} \leqslant\left(\frac{M}{m}\right)^{\frac{1}{p q}} \int_{X} f g d \mu .
$$

Definition 1.1. A function $I \subseteq R \rightarrow R$ is said to be convex (concave) if whenever $x, y \in[a, b]$ and $t \in[0,1]$, the following inequality holds:

$$
f(t x+(1-t) y) \leqslant(\geq) t f(x)+(1-t) f(y) .
$$

Anderson mentioned mean function in [4] as follows:

Definition 1.2. A function $M:(0, \infty) \rightarrow(0, \infty)$ is called a mean function if

(a) Symmetry: $M(x, y)=M(y, x)$;

(b) Reflexivity: $M(x, x)=x$;

(c) Monotonicity: $\min \{x, y\} \leqslant M(x, y) \leqslant \max \{x, y\}$;

(d) Homogeneity: $M(\lambda x, \lambda y)=\lambda M(x, y)$, for any positive scalar $\lambda$.

Definition 1.3. Let $I \rightarrow(0, \infty)$ be continuous, where $I$ is a subinterval of $(0, \infty)$. Let $M$ and $N$ be any two mean functions. We say $f$ is $M N$-convex (concave) if $f(M(x, y)) \leqslant(\geqslant) N(f(x), f(y))$ for all $x, y \in I$.

Taking into account Definition 1.3, $M N$-convex function will be defined by the formulas:

1. $f$ is $A A$-convex iff (1.4) holds;

2. $f$ is $A G$-convex iff

$$
f(t \alpha+(1-t)) \leqslant[f(\alpha)]^{t}[f(\beta)]^{t}, \quad 0 \leqslant t \leqslant 1 ;
$$


3. $f$ is $A H$-convex iff

$$
f((1-t) \alpha+t \beta) \leqslant \frac{f(\alpha) f(\beta)}{t f(\alpha)+(1-t) f(\beta)}, \quad 0 \leqslant t \leqslant 1 ;
$$

4. $f$ is $G A$-convex iff

$$
f\left(\alpha^{t} \beta^{1-t}\right) \leqslant t f(\alpha)+(1-t) f(\beta), \quad 0 \leqslant t \leqslant 1 ;
$$

5. $f$ is $G G$-convex iff

$$
f\left(\alpha^{t} \beta^{1-t}\right) \leqslant[f(\alpha)]^{t}[f(\beta)]^{1-t}, \quad 0 \leqslant t \leqslant 1 ;
$$

6. $f$ is $G H$-convex iff

$$
f\left(\alpha^{1-t} \beta^{t}\right) \leqslant \frac{f(\alpha) f(\beta)}{t f(\alpha)+(1-t) f(\beta)}, \quad 0 \leqslant t \leqslant 1 ;
$$

7. $f$ is $H A$-convex iff

$$
f\left(\frac{\alpha \beta}{(1-t) \alpha+t \beta}\right) \leqslant t f(\alpha)+(1-t) f(\beta), \quad 0 \leqslant t \leqslant 1 ;
$$

8. $f$ is $H G$-convex iff

$$
f\left(\frac{\alpha \beta}{(1-t) \alpha+t \beta}\right) \leqslant[f(\alpha)]^{t}[f(\beta)]^{1-t}, \quad 0 \leqslant t \leqslant 1 ;
$$

9. $f$ is $H H$-convex iff

$$
f\left(\frac{\alpha \beta}{(1-t) \alpha+t \beta}\right) \leqslant \frac{f(\alpha) f(\beta)}{(1-t) f(\alpha)+t f(\beta)}, \quad 0 \leqslant t \leqslant 1 .
$$

The main aim of this paper is to prove some new Wirtinger-type integral inequalities for convex and $M N$-convex functions.

\section{Main Results}

Theorem 2.1. Let $f$ and $f^{\prime}$ be continuous functions on the interval $(a, b)$, with $a<b, f(a)=f(b)$ and $\int_{a}^{b} f(x) d x=0$. If $\left(f^{\prime}\right)^{2}$ is convex on $[a, b]$, then

$$
\int_{a}^{b}[f(x)]^{2} d x \leqslant \frac{(b-a)^{3}}{(2 \pi)^{2}} \frac{\left[f^{\prime}(a)\right]^{2}+\left[f^{\prime}(b)\right]^{2}}{2}
$$


Proof: Since $\left(f^{\prime}\right)^{2}$ is a convex function on $[a, b]$, therefore for $t \in[0,1]$ we have

$$
\begin{aligned}
& \frac{1}{b-a} \int_{a}^{b}\left[f^{\prime}(x)\right]^{2} d x=\int_{0}^{1}\left[f^{\prime}(t a+(1-t) b)\right]^{2} d t \\
& \quad \leqslant \int_{0}^{1}\left[t\left[f^{\prime}(a)\right]^{2}+(1-t)\left[f^{\prime}(b)\right]^{2}\right] d t=\frac{\left[f^{\prime}(a)\right]^{2}+\left[f^{\prime}(b)\right]^{2}}{2} .
\end{aligned}
$$

Now multiplying both sides of the above inequality by $\frac{(b-a)^{3}}{(2 \pi)^{2}}$ and with (1.2), we get the desired inequality in (2.1).

Theorem 2.2. Let $f$ and $f^{\prime}$ be continuous functions on the interval $(a, b)$, with $a<b, f(a)=f(b)$ and $\int_{a}^{b} f(x) d x=0$. If $f^{\prime}$ is convex on $[a, b]$, then

$$
\int_{a}^{b}[f(x)]^{2} d x \leqslant \frac{(b-a)^{3}}{(2 \pi)^{2}}\left\{\frac{\left(f^{\prime}(a)\right)^{2}+\left(f^{\prime}(a)\right)\left(f^{\prime}(a)\right)+\left(f^{\prime}(b)\right)^{2}}{3}\right\}
$$

Proof: We have

$$
\begin{gathered}
\left(\frac{b-a}{2 \pi}\right)^{2} \int_{a}^{b}\left[f^{\prime}(x)\right]^{2} d x=\frac{(b-a)^{3}}{(2 \pi)^{2}} \int_{0}^{1}\left[f^{\prime}(t a+(1-t) b)\right]^{2} d t \\
\leqslant \frac{(b-a)^{3}}{(2 \pi)^{2}} \int_{0}^{1}\left[t f^{\prime}(a)+(1-t) f^{\prime}(b)\right]^{2} d t \\
=\frac{(b-a)^{3}}{(2 \pi)^{2}}\left\{\frac{\left(f^{\prime}(a)\right)^{2}+\left(f^{\prime}(a)\right)\left(f^{\prime}(a)\right)+\left(f^{\prime}(b)\right)^{2}}{3}\right\} .
\end{gathered}
$$

By applying (1.2), we get (2.2).

Theorem 2.3. Let $f$ and $f^{\prime}$ be continuous functions on the interval $(a, b)$ with $a<b, f(a)=f(b)$ and $\int_{a}^{b} f(x) d x=0$. If $f^{\prime}$ is positive, $\left(f^{\prime}\right)^{\frac{1}{\alpha}}$ and $\left(f^{\prime}\right)^{\frac{1}{\beta}}$ are convex on $[a, b]$, then the following inequality holds

$\left.\int_{a}^{b} 2\left[\begin{array}{l}b \\ 0\end{array}\right)(x)\right]^{2} d x \leqslant \alpha(b-a)^{3} \frac{\left[f^{\prime}(a)\right]^{\frac{1}{\alpha}}+\left[f^{\prime}(b)\right]^{\frac{1}{\alpha}}}{8 \pi^{2}}+\beta(b-a)^{3} \frac{\left[f^{\prime}(a)\right]^{\frac{1}{\beta}}+\left[f^{\prime}(b)\right]^{\frac{1}{\beta}}}{8 \pi^{2}}$, where $\alpha, \beta>0$ and $\alpha+\beta=1$. 
Proof: By using the well-known inequality $c d \leqslant \alpha c^{\frac{1}{\alpha}}+\beta d^{\frac{1}{\beta}}(\alpha, \beta, c, d>0$ and $\alpha+\beta=1)$, the convexity of $\left(f^{\prime}\right)^{\frac{1}{\alpha}}$ and $\left(f^{\prime}\right)^{\frac{1}{\beta}}$, we get

$$
\begin{aligned}
& \left(\frac{b-a}{2 \pi}\right)^{2} \int_{a}^{b}\left[f^{\prime}(x)\right]^{2} d x=\frac{(b-a)^{3}}{(2 \pi)^{2}} \int_{0}^{1} f^{\prime}(t a+(1-t) b) f^{\prime}(t a+(1-t) b) d t \\
& \leqslant \frac{(b-a)^{3}}{(2 \pi)^{2}}\left\{\alpha \int_{0}^{1}\left[f^{\prime}(t a+(1-t) b)\right]^{\frac{1}{\alpha}} d t+\beta \int_{0}^{1}\left[f^{\prime}(t a+(1-t) b)\right]^{\frac{1}{\beta}} d t\right\} \\
& \leqslant \frac{(b-a)^{3}}{(2 \pi)^{2}}\left\{\alpha \int_{0}^{1}\left[t f^{\prime}(a)+(1-t) f^{\prime}(b)\right]^{\frac{1}{\alpha}} d t+\beta \int_{0}^{1}\left[t f^{\prime}(a)+(1-t) f^{\prime}(b)\right]^{\frac{1}{\beta}} d t\right\} \\
& \leqslant \frac{(b-a)^{3}}{(2 \pi)^{2}}\left\{\alpha \int_{0}^{1}\left[t\left(f^{\prime}(a)\right)^{\frac{1}{\alpha}}+(1-t)\left(f^{\prime}(b)\right)^{\frac{1}{\alpha}}\right] d t\right. \\
& \left.+\beta \int_{0}^{1}\left[t\left(f^{\prime}(a)\right)^{\frac{1}{\beta}}+(1-t)\left(f^{\prime}(b)\right)^{\frac{1}{\alpha}}\right] d t\right\} \\
& =\frac{(b-a)^{3}}{(2 \pi)^{2}}\left\{\alpha \frac{\left(f^{\prime}(a)\right)^{\frac{1}{\alpha}}+\left(f^{\prime}(b)\right)^{\frac{1}{\alpha}}}{2}+\beta \frac{\left(f^{\prime}(a)\right)^{\frac{1}{\beta}}+\left(f^{\prime}(b)\right)^{\frac{1}{\beta}}}{2}\right\} .
\end{aligned}
$$

Combining with (1.2), we get the required inequality.

Theorem 2.4. Let $f$ and $f^{\prime}$ be continuous on the interval $(a, b)$, with $a<b$, $f(a)=f(b), \int^{b} f(x) d x=0$ and $f>0$. Let $0<m \leqslant \frac{|f|^{p}}{|f|^{q}} \leqslant M<\infty$ for $p, q>1$ with $\frac{1}{p}+\frac{1}{q}=1$. If $|f|^{p},|f|^{q}$ are concave on $[a, b]$ then

$$
\left(\frac{m}{M}\right)^{\frac{1}{p q}}[f(a)+f(b)]^{2} \leqslant \frac{b-a}{\pi^{2}} \int_{a}^{b}\left[f^{\prime}(x)\right]^{2} d x .
$$

Proof: Making changes to the variable, using the reverse Hölder inequality and inequality $|u+v|^{r} \leqslant 2^{r-1}\left(|u|^{r}+|v|^{r}\right), u, v \in \mathbb{R}$, we have

$$
\begin{aligned}
& \frac{1}{b-a} \int_{a}^{b}[f(x)]^{2} d x=\int_{0}^{1}[f(t a+(1-t) b)]^{2} d t \\
& \geqslant\left(\frac{m}{M}\right)^{\frac{1}{p q}}\left(\int_{0}^{1}|f(t a+(1-t) b)|^{p} d t\right)^{\frac{1}{p}}\left(\int_{0}^{1}|f(t a+(1-t) b)|^{q} d t\right)^{\frac{1}{q}}
\end{aligned}
$$




$$
\begin{aligned}
\geqslant & \left(\frac{m}{M}\right)^{\frac{1}{p q}}\left(\int_{0}^{1}\left[t|f(a)|^{p}+(1-t)|f(b)|^{p}\right] d t\right)^{\frac{1}{p}} \\
& \cdot\left(\int_{0}^{1}\left[t|f(a)|^{q}+(1-t)|f(b)|^{q}\right] d t\right)^{\frac{1}{q}} \\
= & \left(\frac{m}{M}\right)^{\frac{1}{p q}}\left(\frac{|f(a)|^{p}+|f(b)|^{p}}{2}\right)^{\frac{1}{p}}\left(\frac{|f(a)|^{q}+|f(b)|^{q}}{2}\right)^{\frac{1}{q}} \\
\geqslant & \left(\frac{m}{M}\right)^{\frac{1}{p q}}\left(\frac{|f(a)+f(b)|^{p}}{2^{p}}\right)^{\frac{1}{p}}\left(\frac{|f(a)+f(b)|^{q}}{2^{q}}\right)^{\frac{1}{q}} \\
= & \left(\frac{m}{M}\right)^{\frac{1}{p q}} \frac{(f(a)+f(b))^{2}}{4} .
\end{aligned}
$$

By (1.2), we get the inequality (2.4).

Theorem 2.5. Let $f$ and $f^{\prime}$ be continuous on the interval $(a, b)$, with $a<b$, $f(a)=f(b)$ and $\int_{a}^{b} f(x) d x=0$. Then:

1. If $\left|f^{\prime}\right|$ is $A G$ convex, then

$$
\int_{a}^{b}[f(x)]^{2} d x \leqslant \frac{(b-a)^{3}}{8 \pi^{2}} \frac{\left[f^{\prime}(a) f^{\prime}(b)\right]^{2}-1}{\ln \left[f^{\prime}(a) f^{\prime}(b)\right]} ;
$$

2. If $\left|f^{\prime}\right|$ is AH convex, then

$$
\int_{a}^{b}[f(x)]^{2} d x \leqslant \frac{(b-a)^{3}}{(2 \pi)^{2}} f^{\prime}(a) f^{\prime}(b) ;
$$

3. If $\left|f^{\prime}\right|$ is GA convex, then

$$
\begin{array}{rl}
\int_{a}^{b}[f(x)]^{2} & d x \leqslant\left(\frac{b-a}{2 \pi}\right)^{2}\left\{\left[-a+\frac{2 a}{\ln \frac{a}{b}}+2 \frac{b-a}{\ln ^{2} \frac{a}{b}}\right]\left[f^{\prime}(a)\right]^{2}\right. \\
+ & {\left[-2(a+b) \frac{1}{\ln \frac{a}{b}}-4 \frac{b-a}{\ln ^{2} \frac{a}{b}}\right] f^{\prime}(a) f^{\prime}(b)} \\
+ & \left.\left[b+\frac{2 b}{\ln \frac{a}{b}}+\frac{2(b-a)}{\ln ^{2} \frac{a}{b}}\right]\left[f^{\prime}(b)\right]^{2}\right\}
\end{array}
$$


4. If $\left|f^{\prime}\right|$ is $G G$ convex, then

$$
\int_{a}^{b}[f(x)]^{2} d x \leqslant\left(\frac{b-a}{2 \pi}\right)^{2} \ln \frac{a}{b} \frac{b\left[f^{\prime}(b)\right]^{2}-a\left[f^{\prime}(a)\right]^{2}}{\ln a\left[f^{\prime}(a)\right]^{2}-\ln b\left[f^{\prime}(b)\right]^{2}}
$$

5. If $\left|f^{\prime}\right|$ is $H A$ convex, then

$$
\begin{array}{rl}
\int_{a}^{b}[f(x)]^{2} & d x \leqslant \frac{1}{(2 \pi)^{2}}\left\{\left[a(b-a)\left(b+a(b-a)^{2}\right)-a^{2} b \ln \frac{b}{a}\right]\left[f^{\prime}(a)\right]^{2}\right. \\
+ & {\left[a b(b-a)\left(1-(b-a)^{2}\right)-a b(a+b) \ln \frac{b}{a}\right] f^{\prime}(a) f^{\prime}(b)} \\
+ & \left.\left[b(b-a)\left(a+b(b-a)^{2}\right)-a b^{2} \ln \frac{b}{a}\right]\left[f^{\prime}(b)\right]^{2}\right\} .
\end{array}
$$

Proof.

1. From (1.2) and by using the $A G$ convexity of $\left|f^{\prime}\right|$ we have

$$
\begin{aligned}
\int_{a}^{b}[f(x)]^{2} d x \leqslant\left(\frac{b-a}{2 \pi}\right)^{2} \int_{a}^{b}\left[f^{\prime}(x)\right]^{2} d x=\frac{(b-a)^{3}}{(2 \pi)^{2}} \int_{0}^{1}\left[f^{\prime}(t a+(1-t) b)\right]^{2} d t \\
\quad \leqslant \frac{(b-a)^{3}}{(2 \pi)^{2}} \int_{0}^{1}\left[\left(f^{\prime}(a)\right)^{t}\left(f^{\prime}(b)\right)^{t}\right]^{2} d t=\frac{(b-a)^{3}}{(2 \pi)^{2}} \int_{0}^{1}\left[\left(f^{\prime}(a)\right)\left(f^{\prime}(b)\right)\right]^{2 t} d t \\
\quad=\frac{(b-a)^{3}}{8 \pi^{2}} \frac{\left[f^{\prime}(a) f^{\prime}(b)\right]^{2}-1}{\ln \left[f^{\prime}(a) f^{\prime}(b)\right]}
\end{aligned}
$$

2. Since $\left|f^{\prime}\right|$ is an $A H$-convex function, we can write

$$
\begin{gathered}
\int_{a}^{b}[f(x)]^{2} d x \leqslant\left(\frac{b-a}{2 \pi}\right)^{2} \int_{a}^{b}\left[f^{\prime}(x)\right]^{2} d x=\frac{(b-a)^{3}}{(2 \pi)^{2}} \int_{0}^{1}\left[f^{\prime}((1-t) a+t b)\right]^{2} d t \\
\leqslant \frac{(b-a)^{3}}{(2 \pi)^{2}} \int_{0}^{1}\left[\frac{f^{\prime}(a) f^{\prime}(b)}{t f^{\prime}(a)+(1-t) f^{\prime}(b)}\right]^{2} d t=\frac{(b-a)^{3}}{(2 \pi)^{2}} f^{\prime}(a) f^{\prime}(b) ;
\end{gathered}
$$


3. Taking into account that $\left|f^{\prime}\right|$ is $G A$ - convex, we have

$$
\begin{aligned}
\int_{a}^{b}[f(x)]^{2} d x \leqslant\left(\frac{b-a}{2 \pi}\right)^{2} \int_{a}^{b}\left[f^{\prime}(x)\right]^{2} d x=\left(\frac{b-a}{2 \pi}\right)^{2} \ln \frac{a}{b} \int_{1}^{0}\left[f^{\prime}\left(a^{t} b^{1-t}\right)\right]^{2} a^{t} b^{1-t} d t \\
\leqslant\left(\frac{b-a}{2 \pi}\right)^{2} \ln \frac{a}{b} \int_{1}^{0}\left[t f^{\prime}(a)+(1-t) f^{\prime}(b)\right]^{2} a^{t} b^{1-t} d t \\
=b\left(\frac{b-a}{2 \pi}\right)^{2} \ln \frac{a}{b}\left\{\left[\left(f^{\prime}(a)\right)^{2}-2 f^{\prime}(a) f^{\prime}(b)+\left(f^{\prime}(b)\right)^{2}\right] \int_{1}^{0} t^{2}\left(\frac{a}{b}\right)^{t} d t\right. \\
\left.+\left[2 f^{\prime}(a) f^{\prime}(b)-2\left(f^{\prime}(b)\right)^{2}\right] \int_{1}^{0} t\left(\frac{a}{b}\right)^{t} d t+\left(f^{\prime}(b)\right)^{2} \int_{1}^{0}\left(\frac{a}{b}\right)^{t} d t\right\} \\
=\left(\frac{b-a}{2 \pi}\right)^{2}\left\{\left[-a+\frac{2 a}{\ln ^{2} \frac{a}{b}}+2 \frac{b-a}{\ln ^{2} \frac{a}{b}}\right]\left[f^{\prime}(a)\right]^{2}\right. \\
+\left[-2(a+b) \frac{1}{\ln ^{\frac{a}{b}}-4} \frac{b-a}{\ln ^{2} \frac{a}{b}}\right] f^{\prime}(a) f^{\prime}(b) \\
\left.+\left[b+\frac{2 b}{\ln \frac{a}{b}}+\frac{2(b-a)}{\ln ^{2} \frac{a}{b}}\right]\left[f^{\prime}(b)\right]^{2}\right\}
\end{aligned}
$$

4. Since $\left|f^{\prime}\right|$ is $G G$-convex, we have

$$
\begin{aligned}
\int_{a}^{b}[f(x)]^{2} d x \leqslant\left(\frac{b-a}{2 \pi}\right)^{2} \int_{a}^{b}\left[f^{\prime}(x)\right]^{2} d x \\
=\left(\frac{b-a}{2 \pi}\right)^{2} \ln \frac{a}{b} \int_{1}^{0}\left[f^{\prime}\left(a^{t} b^{1-t}\right)\right]^{2} a^{t} b^{1-t} d t \\
\quad \leqslant\left(\frac{b-a}{2 \pi}\right)^{2} \ln \frac{a}{b} \int_{1}^{0}\left\{\left[f^{\prime}(a)\right]^{t}\left[f^{\prime}(b)\right]^{1-t}\right\}^{2} a^{t} b^{1-t} d t \\
=b\left(\frac{b-a}{2 \pi}\right)^{2} \ln \frac{a}{b}\left[f^{\prime}(b)\right]^{2} \int_{1}^{0}\left\{\frac{a\left[f^{\prime}(a)\right]^{2}}{b\left[f^{\prime}(b)\right]^{2}}\right\}^{t} d t \\
=\left(\frac{b-a}{2 \pi}\right)^{2} \ln \frac{a}{b} \frac{b\left[f^{\prime}(b)\right]^{2}-a\left(f^{\prime}(a)\right)^{2}}{\ln a\left[f^{\prime}(a)\right]^{2}-\ln b\left[f^{\prime}(b)\right]^{2}} ;
\end{aligned}
$$


5. Since $\left|f^{\prime}\right|$ is $H A$-convex, we have

$$
\begin{aligned}
\int_{a}^{b}[f(x)]^{2} d x \leqslant\left(\frac{b-a}{2 \pi}\right)^{2} \int_{a}^{b}\left[f^{\prime}(x)\right]^{2} d x \\
=a b \frac{(b-a)^{3}}{(2 \pi)^{2}} \int_{0}^{1}\left[f^{\prime}\left(\frac{a b}{(1-t) a+t b}\right)\right]^{2} \frac{1}{[(1-t) a+t b]^{2}} d t \\
\leqslant a b \frac{(b-a)^{3}}{(2 \pi)^{2}} \int_{0}^{1}\left[\left(\frac{t f^{\prime}(a)+(1-t) f^{\prime}(b)}{(1-t) a+t b}\right)\right]^{2} d t \\
=\frac{1}{(2 \pi)^{2}}\left\{\left[a(b-a)\left(b+a(b-a)^{2}\right)-a^{2} b \ln \frac{b}{a}\right]\left[f^{\prime}(a)\right]^{2}\right. \\
+\left[a b(b-a)\left(1-(b-a)^{2}\right)-a b(a+b) \ln \frac{b}{a}\right] f^{\prime}(a) f^{\prime}(b) \\
\left.+\left[b(b-a)\left(a+b(b-a)^{2}\right)-a b^{2} \ln \frac{b}{a}\right]\left[f^{\prime}(b)\right]^{2}\right\} . \square
\end{aligned}
$$

\section{R E F E R E N C E S}

1. W. BlaschKe: Kreis and Kugel, Leipzig, 1916, pp. 105-106.

2. P. BeEsack: Integral inequalities of the Wirtinger type. Duke Math. J. 25, (1958), $477-498$.

3. M. Mitrinović and P. VAsić: An integral inequality ascribed to Wirtinger, and its variations and generalization. Univ. Beograd. Publ. Elektrotehn. Fak. Ser. Mat. Fiz (1969), 247-273.

4. G. D. Anderson, M. K. Vamanamurthy and M. Vuorinen: Generalized convexity and inequalities. J. Math. Anal. Appl. 335, (2007), 1294-1308.

Tatjana Z. Mirković

College of Applied Professional Studies

Filipa Filipovića bb

17000 Vranje, Serbia

tmirkovic75@gmail.com 